\title{
Outcomes of Combined Neck and Trochanter Fractures of the Femur Treated with Cephallomedullary Nail in Elderly
}

\author{
Hyunseung Yoo, MD, Youngho Cho, MD, Seongmun Hwang, MD \\ Department of Orthopaedic Surgery, Daegu Fatima Hospital, Daegu, Korea
}

Purpose: Proximal femur fractures are classified into intracapsular neck fractures and extracapsular trochanteric fractures, and several related treatment recommendations in elderly patients have already been introduced. Importantly, we have observed cases of combined intra and extracapsular fractures (i.e., ipsilateral neck and trochanter fractures). The purpose of this study is to report the outcomes of combined neck and trochanter fractures of the femur treated with cephalomedullary nail (CMN) in elderly patients.

Materials and Methods: From January 2010 to December 2014, 410 patients with proximal femoral fractures were fixed using CMN; among this group, 37 patients with combined neck and trochanter fractures were identified. Two of these patients died fewer than three months after injury and another two did not return for follow-up. Thirty-three patients were included and reviewed retrospectively in this study.

Results: All patients were injured by simple fall. Bone union was obtained in 28 of 33 patients. Of the five patients who failed treatment, three experienced implant penetration through head (cut-through and cut-out), one had breakage of CMN and the last one had a loosening of internal fixation device with persistent non-union at final follow-up. The former four patients underwent hip replacement surgery and the latter refused surgery because he had low demand in daily life and many medical problems.

Conclusion: Eighty-five percent of elderly patients with combined neck and trochanter fractures of the femur treated with $\mathrm{CMN}$ achieved bone union; these complex fractures require more accurate reduction than usual extra-articular intertrochanteric fractures.

Key Words: Femur, Neck and trochanter fracture, Cephalomedullary nail

Submitted: June 26, 2019 1st revision: August 1, 2019

2nd revision: August 31, 2019 Final acceptance: September 19, 2019

Address reprint request to

Youngho Cho, MD

[https://orcid.org/0000-0002-1527-7761]

Department of Orthopaedic Surgery, Daegu Fatima Hospital, 99

Ayang-ro, Dong-gu, Daegu 41199, Korea

TEL: +82-53-940-7320 FAX: +82-53-954-7417

E-mail: femur1973dagmail.com

This is an Open Access article distributed under the terms of the Creative Commons Attribution Non-Commercial License (http://creativecommons. org/licenses/by-nc/4.0) which permits unrestricted non-commercial use, distribution, and reproduction in any medium, provided the original work is properly cited.

\section{INTRODUCTION}

Despite the continued optimization of treatments for osteoporosis over the last decade, the absolute incidence of hip fractures in elderly patients is increasing ${ }^{1}$. The classic treatment approach for hip fracture in elderly is as follows: i) osteosynthesis (preferred treatment for exrtracapsular trochanteric fractures) and ii) arthroplasty (an easy treatment for displaced femoral neck fractures such as intracapsular fractures).

Dynamic hip screws (DHS) have been shown to be very effective for the surgical treatment of acute 
basicervical fractures or stable trochanteric fractures of femur by closed reduction and internal fixation ${ }^{2,3}$. In cases of unstable intertrochanteric fractures, a surgery is challenging because of the loss of medial buttress and lateral wall fractures ${ }^{4}$; some surgeons have attempted hip arthroplasty for this unstable fracture and experienced satisfactory results ${ }^{5-8}$. However, osteosynthesis is still the standard treatment for most trochanteric fractures regardless of the comminution and degree of osteoporosis. Internal fixation with cephalomedullary nail (CMN) and DHS with trochanter stabilizing plate (TSP) have led to satisfactory results for the treatment of unstable intertrochanteric fractures ${ }^{9-12}$.

Combined neck and trochanteric fractures, the focus of the current study, do not belong to any classification system. In other words, proximal main fracture fragment is fractured through femoral neck, and there were several case reports about treating this unique fracture ${ }^{13-15)}$. The purpose of the current study was to report the outcomes of combined neck and trochanter fractures of the femur treated with CMN in elderly patients.

\section{MATERIALS AND METHODS}

Between January 2010 and December 2014, a retrospective radiographic review was conducted of all patients treated with $\mathrm{CMN}$ of a proximal femoral fracture. During this period, 410 patients with proximal femoral fractures were fixed using CMN. Patients were included

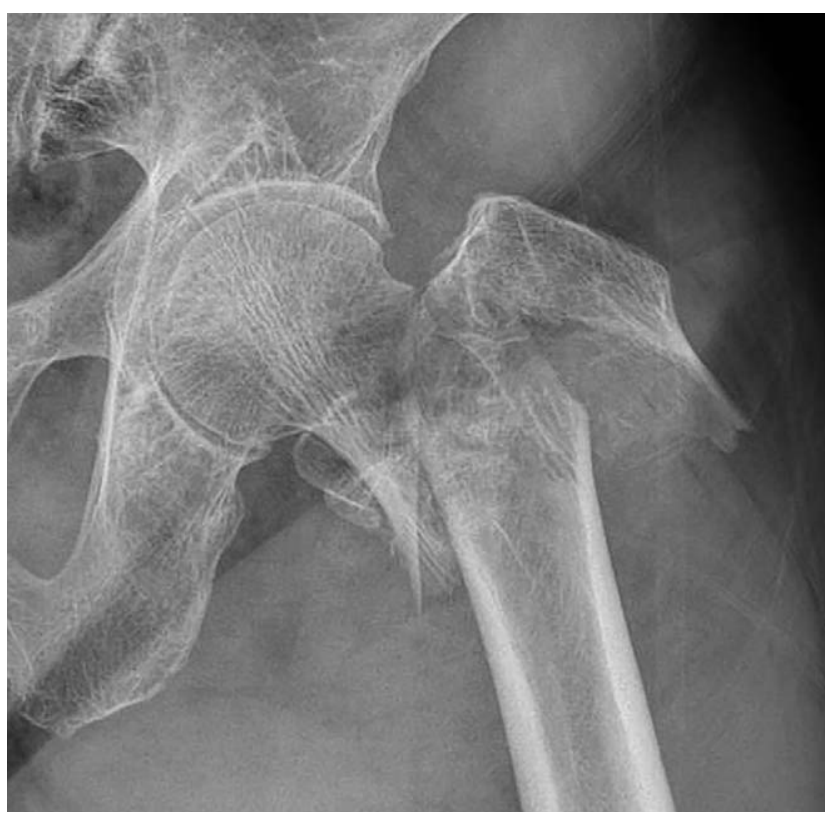

Fig. 1. Anteroposterior radiographs of 4 part fracture. in the current study if preoperative radiographs revealed combined neck and trochanter fracture of the proximal part of the femur (i.e., three or four fractures have separated fragments). Each fragment was a greater trochanter fracture, lesser trochanter fracture, distal fragment and neck fracture of the proximal femur (Fig. 1). Fractures with a fracture line exiting distal to the upper border of lesser trochanter were excluded. We also reviewed all intraoperative fluoroscopic images to ensure the neck fracture. Overall, 37 of 410 patients met the inclusion criteria; two of these patients died fewer than three months after injury and another two patients did not return for follow-up. Finally, we review 33 patients in this study. Table 1 presents the basic demographic data of patients of this study.

All patients underwent surgery using proximal femoral nail antirotation II (PFNA II; Synthes, Oberdorf, Switzerland). Fracture reduction and fixation was attempted by standard method on the fracture table under fluoroscopic guide. A bone hook was occasional used for reduction and a Steinmann pin was inserted for the maintenance of temporary reduction during the fixation of implants. Sitting and wheelchair ambulation was encouraged immediately after surgery.

The tip-apex distance (TAD) is the sum of the distance in millimeters from the tip of the lag screw to the apex of the femoral head as measured on an anteroposterior (AP) radiograph and that distance on a lateral radiograph, after correction for magnification ${ }^{16}$. We calculated it on immediate postoperative radiographs.

Institutional review board approval was obtained for this study (Daegu Fatima Hospital; IRB No. DFE19ORIO 044-R1).

Statistical analyses were conducted with the SPSS ver.

Table 1. Basic Demographic Data

\begin{tabular}{lc}
\hline \hline Variable & Value \\
\hline Age (yr) & $77(61-90)$ \\
Sex (female/male) & $29 / 4$ \\
Follow-up (mo) & $21.8(6-63)$ \\
Bone mineral density at & $-3.2(-5.3--0.56)$ \\
femur neck (T-score) & \\
Fracture type* & 20 \\
3 part & 13 \\
4 part & \\
\hline
\end{tabular}

Values are presented as median (range) or number only.

* Depends on the number of fracture fragment. 3 part fracture have a greater trochanter (GT), neck and distal fragment. 4 part fracture have a GT, lesser trochanter, neck and distal fragment. 


\section{Hip \& Pelvis}

Hip Pelvis 31(4): 200-205, 2019

16.0 (SPSS Inc., Chicago, IL, USA). For all other tests, a two-sided $P$-value $<0.05$ was considered significant.

\section{RESULTS}

A total of 33 patients were injured by simple fall (i.e., fall from a standing height). TAD was less than $25 \mathrm{~mm}$ in all patients. According to our own classification, threepart fractures (neck and greater trochanter fracture) occurred in 20 cases and four-part fractures (neck, greater and lesser trochanter fracture) occurred in 13. All patients had separated greater trochanter fracture fragment that was confirmed by preoperative computed tomogram scan.

Twenty-eight patients experienced an uneventful bone union and five experienced treatment failure (i.e., breakage of implant, movement of helical blade out of the femoral head, or nonunion of the fracture). Three patients had penetration of implant through the head (cut-through and
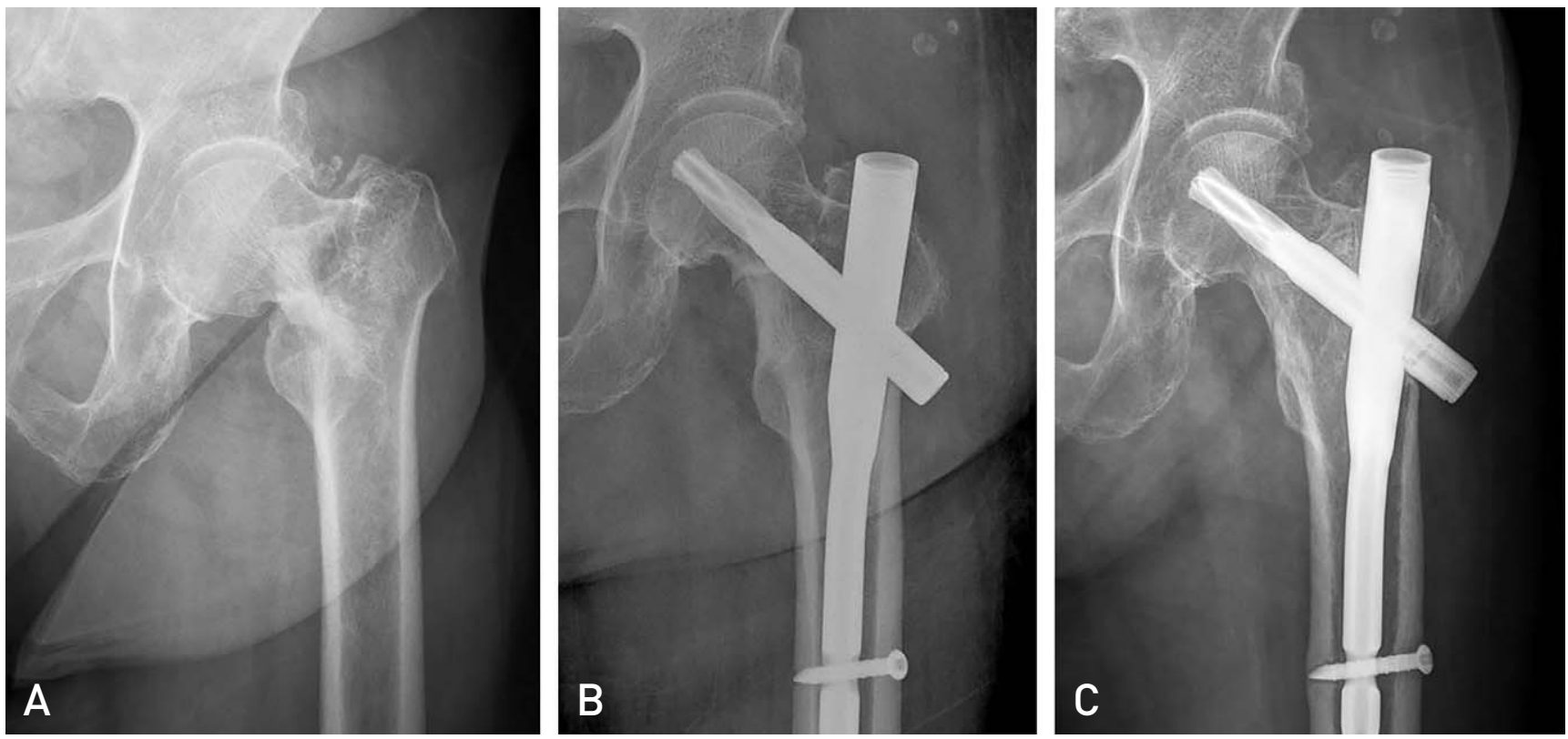

Fig. 2. (A) Anteroposterior radiograph shows the neck and greater trochanter fracture. (B) Immediate postoperative X-ray shows anatomic reduction for femoral neck fracture. (C) Five year follow-up X-ray shows solid bone union.
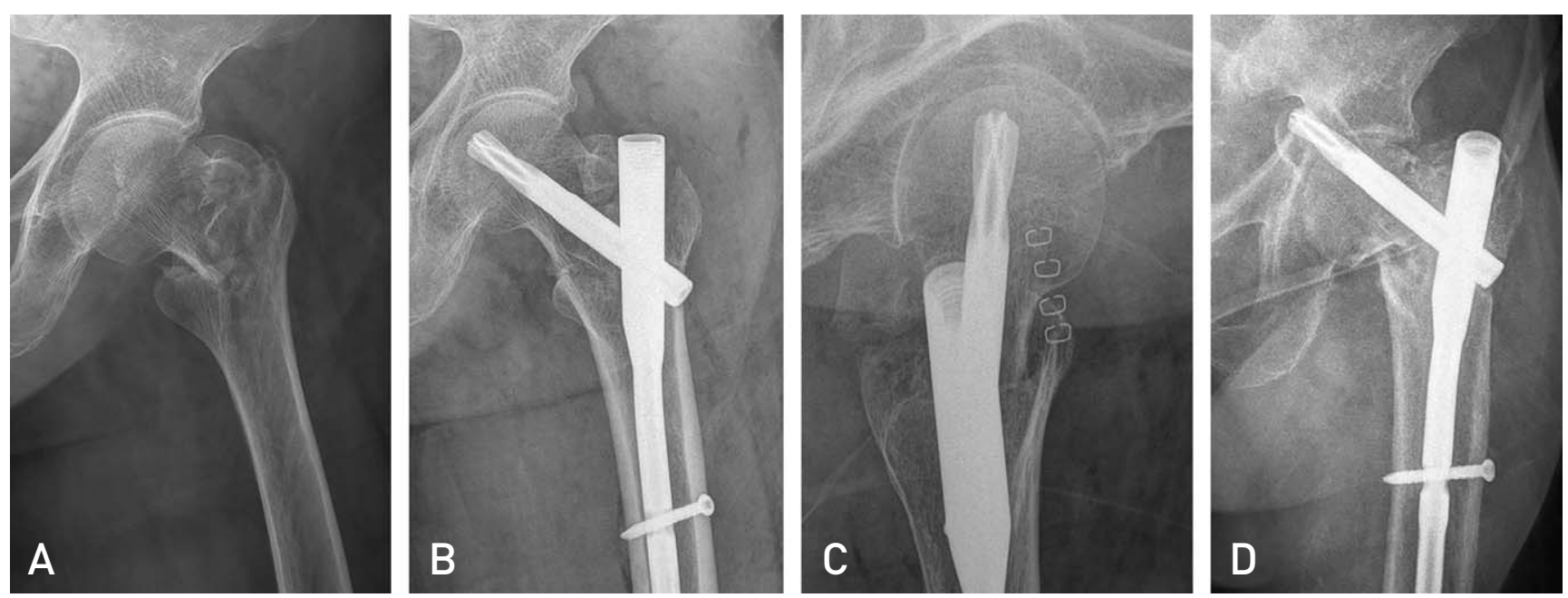

Fig. 3. (A) Anteroposterior (AP) X-ray shows the basal neck and greater trochanter fracture. (B) Immediate postoperative Xray shows anatomic alignment on AP view and intra-medullary reduction on lateral view. (C) Immediate postoperative X-ray shows anatomic alignment on AP view and intra-medullary reduction on lateral view. (D) Sixteen months follow-up X-ray shows penetration of blade out of femoral head. 
Hip\& Pelvis

Hyunseung Yoo et al. Neck and Trochanter Fractures

Table 2. Summary of Failed Cases

\begin{tabular}{ccccccc}
\hline \hline & Sex/Age (yr) & Fracture pattern & Failure time $(\mathrm{mo})$ & BMD of femur neck $\left(\mathrm{g} / \mathrm{cm}^{2}\right)$ & Mode of failure & Revision \\
\hline 1 & Female/73 & 4 part & 31 & -0.56 & Implant fracture & BHA \\
2 & Female/88 & 3 part & 28 & -2.9 & Cut through & BHA \\
3 & Female/81 & 4 part & 6 & -3.4 & Cut out & BHA \\
4 & Female/84 & 3 part & 19 & -3.5 & Cut through & BHA \\
5 & Male/82 & 4 part & - & -4.4 & Implant loosening \\
\hline
\end{tabular}

BMD: bone mineral density, BHA: bipolar hemiarthroplasty.

cut-out), one had breakage of CMN and the last one had a loosening of internal fixation device with persistent nonunion at the end of follow-up.

There was no difference in BMD between the union group and the non-union group $(P>0.05)$. According to the fracture pattern, two out of 20 patients failed in threepart fractures $(10.0 \%)$ and three of 13 patients failed in four-part fractures $(23.1 \%)$. Though there were more nonunion patients in the four-part fracture group, we found no statistical significance between fracture patterns. Patients who had the anatomical reduction or anatomical alignment on both AP and lateral radiographs between neck fragment and distal shaft fragment could achieve bone union (Fig. 2). All five patients who failed showed satisfactory reduction in AP view but not in the lateral view. Every lateral radiograph revealed posterior angulation or posterior sagging of the neck fragment (Fig. 3).

Table 2 summarizes all failed cases. Four patients were revised with bipolar hemiarthroplasty after fixation failure. The final patient had refused revision surgery until 49 months after the index surgery because he had a little pain, low demand in daily life and many medical problems.

\section{DISCUSSION}

An AO/OTA 31A fracture refers to an extracapsular fracture (i.e., pertrochanter or intertrochanter fracture), while $31 \mathrm{~B}$ refers to an intracapsular neck fracture. Combined neck and trochanter fractures of the femur are not currently classified. Some surgeons consider these fractures basicervical comminution, while others consider them a variant of pertrochanter fractures. Importantly, these fracture types are not uncommon and most surgeons have at least come experience with partially intracapsular (proximal main fragment) and partially extracapsular (greater and lesser trochanter fragment) fractures. It is important to note that the proximal main fragment is enveloped by a joint capsule, made up of anterior and medial portions that are critically important for stability after fixation. Because the femoral neck does not have a cambium layer of periosteum, patients are unable to achieve secondary callus-forming bone healing. In other words, the proximal main neck fragment can be healed by a primary bone healing that doesn't make callus. Bone to bone contact in the anteromedial cortex is essential for bone union.

Combined neck and trochanter fracture is an unstable fracture, because multiple fracture fragment, rotational instability of neck fragment and occult lateral wall fracture. Treatment is more complex than osteosynthesis for trochanteric fracture and arthroplasty for displaced femoral neck fracture. Some surgeons had tried hip arthroplasty for unstable trochanteric fracture and showed satisfactory results ${ }^{5-8)}$. However, arthroplasty for unstable trochanteric fracture results in longer operation time, more blood loss and transfusion volume than arthroplasty for displaced femoral neck fractures.

Common fixation devices for proximal femoral fracture are DHS and CMN. A DHS had shown to be very effective in the surgical treatment of acute basicervical fractures or stable trochanteric fractures of femur by closed reduction and internal fixation ${ }^{2,3}$. Some additional implant such as TSP could be applied for unstable trochanteric fractures ${ }^{9-11}$.

Isaacs and Lawrence ${ }^{13)}$ reported concomitant ipsilateral intertrochanteric and subcapital fracture of the hip treated with DHS and didn't give a final result because patient died from complication of cancer shortly after discharge. Kumar et al. ${ }^{14)}$ reported similar case treated with DHS and gained satisfactory healing with minimal collapse of the head at one year following injury. Perry and Scott ${ }^{15)}$ reported the concomitant ipsilateral intracapsular and extracapsular femoral neck fracture treated with DHS in geriatric patient and they unfortunately experienced the fixation failure. As you have seen, the results of the treatment with DHS are varying.

There are several reports the treatment of basicervical fractures of the femur with $\mathrm{CMN}^{17-20)}$. The success rate of 
treatment with CMN is wide range, from $45 \%$ to $100 \%$. Watson et al. ${ }^{18)}$ reported CMN may be inadequate for fixation of 2-part basicervical fractures as a result of the instability and orientation of basicervical fractures. They concluded that these fracture may be better treated with a compression hip screw and plate device. However, they didn't give a clear explanation for the failure.

On the other hand, Kweon et al. ${ }^{19)}$ reported CMN fixation for basicervical femoral fractures in elderly showed satisfactory results, considered to be a useful method if performed with skilled technique.

With the recent development of a variety of intramedullary devices, intramedullary nails are less invasive than DHS, which may result in shorter operation time and smaller transfusion volume, and have shown favorable clinical results $^{20)}$.

We treated all our patients with the PFNA II (Synthes). PFNA II was made for Asian people who have a smaller femur than western patients. Its mediolateral angle is reduced to $5^{\circ}$, allowing a slightly more lateral entry point through the tip of the greater trochanter. Furthermore, it has a more flattened lateral surface that theoretically decreases the length of the region of impingement on the lateral cortex. Insertion of the blade compacts the cancellous bone. These characteristics provide optimal anchoring and stability when the implant is inserted into osteoporotic bone and have been bio-mechanically proven to retard rotation and varus collapse. The inserted PFNA blade achieves an excellent fit through bone compaction and requires less bone removal compared to a screw. Improved design of PFNA II used to treat unstable trochanter fracture reduced complications related implant itself $^{21,22)}$.

During the study period, 37 out of the 410 patients $(9.0 \%)$ treated with PFNA II presented with combined neck and trochanter fractures. Four patients did not complete the required follow-up and another five failed to achieve bone union, thus the failure rate reported here $(15.2 \%)$ is significantly higher than other analyses of unstable trochanteric fracture ${ }^{23)}$. We believe that: i) many trochanteric fracture failures may actually be combined neck and trochanter fractures and ii) surgeons should carefully evaluate the preoperative X-ray and intraoperative fluoroscopic findings to determine whether the proximal fragment is intracapsular or extracapsular.

All failure cases described in this report presented with satisfactory reduction in the AP but not lateral views. Every lateral radiograph revealed posterior angulation or posterior sagging of the neck fragment. Tsukada et al. ${ }^{24)}$ reported posteriorly displaced proximal fragments for pertrochanteric fracture on lateral radiographs slipped more significantly compared with anteriorly displaced or reduced proximal fragments. However, every patient in this analysis had a fracture across the femoral neck. In the current study, we considered that the proximal fragment should be reduced anatomically on the AP and lateral views as the proximal main fragment must be healed without callus formation. Therefore, we recommend that surgeons carefully check the reduction status on both AP and lateral plane radiographs. Whether extramedullary reduction can increase the rate of solid bone union in combined neck and trochanter fractures should be the focus of a future study.

The limitations of our study include sample size (i.e., small number of patients), the retrospective study and that the results are from the experience of a single surgeon. A future study is needed to better classify and analyze optimal treatment approaches for combined neck and trochanter fractures.

\section{CONCLUSION}

Eighty-five percent of elderly patients with combined neck and trochanter fractures of the femur treated with CMN experienced bone union. These complex fractures require more accurate reduction than usual extra-articular intertrochanteric fractures.

\section{CONFLICT OF INTEREST}

The authors declare that there is no potential conflict of interest relevant to this article.

\section{REFERENCES}

1.Lee YK, Kim JW, Lee MH, Moon KH, Koo KH. Trend in the age-adjusted incidence of hip fractures in South Korea: systematic review. Clin Orthop Surg. 2017;9:420-3.

2. Chen CY, Chiu FY, Chen CM, Huang CK, Chen WM, Chen TH. Surgical treatment of basicervical fractures of femur--a prospective evaluation of 269 patients. J Trauma. 2008;64:427-9.

3. Cobelli NJ, Sadler AH. Ender rod versus compression screw fixation of hip fractures. Clin Orthop Relat Res. 1985; (201):123-8.

4.Palm H, Jacobsen S, Sonne-Holm S, Gebuhr P; Hip Fracture Study Group. Integrity of the lateral femoral wall in intertrochanteric hip fractures: an important predictor of a reoperation. J Bone Joint Surg Am. 2007;89:470-5.

5. Kim YS, Hur JS, Hwang KT, Choi IY, Kim YH. The 
comparison of compression hip screw and bipolar hemiarthroplasty for the treatment of AO type A2 intertrochanteric fractures. Hip Pelvis. 2014;26:99-106.

6. Chu X, Liu F, Huang J, Chen L, Li J, Tong P. Good shortterm outcome of arthroplasty with Wagner SL implants for unstable intertrochanteric osteoporotic fractures. J Arthroplasty. 2014;29:605-8.

7. Chan KC, Gill GS. Cemented hemiarthroplasties for elderly patients with intertrochanteric fractures. Clin Orthop Relat Res. 2000;(371):206-15.

8. Rodop O, Kiral A, Kaplan H, Akmaz I. Primary bipolar hemiprosthesis for unstable intertrochanteric fractures. Int Orthop. 2002;26:233-7.

9. Babst R, Renner N, Biedermann M, et al. Clinical results using the trochanter stabilizing plate (TSP): the modular extension of the dynamic hip screw $(\mathrm{DHS})$ for internal fixation of selected unstable intertrochanteric fractures. J Orthop Trauma. 1998;12:392-9.

10. Gupta RK, Sangwan K, Kamboj P, Punia SS, Walecha P. Unstable trochanteric fractures: the role of lateral wall reconstruction. Int Orthop. 2010;34:125-9.

11. Radford PJ, Needoff M, Webb JK. A prospective randomised comparison of the dynamic hip screw and the gamma locking nail. J Bone Joint Surg Br. 1993;75:789-93.

12. Papasimos S, Koutsojannis CM, Panagopoulos A, Megas $\mathrm{P}$, Lambiris E. A randomised comparison of AMBI, TGN and PFN for treatment of unstable trochanteric fractures. Arch Orthop Trauma Surg. 2005;125:462-8.

13. Isaacs C, Lawrence B. Concomitant ipsilateral intertrochanteric and subcapital fracture of the hip. J Orthop Trauma. 1993; 7:146-8.

14. Kumar R, Khan R, Moholkar K, Smyth H, Borton D. A rare combination fracture of the neck of femur. Eur J Orthop Surg Traumatol. 2001;11:59-61.

15. Perry DC, Scott SJ. Concomitant ipsilateral intracapsular and extracapsular femoral neck fracture: a case report. J Med Case Rep. 2008;2:68.

16. Baumgaertner MR, Curtin SL, Lindskog DM, Keggi JM. The value of the tip-apex distance in predicting failure of fixation of peritrochanteric fractures of the hip. $J$ Bone Joint Surg Am. 1995;77:1058-64.

17. Saarenpää I, Partanen J, Jalovaara P. Basicervical fracture--a rare type of hip fracture. Arch Orthop Trauma Surg. 2002; 122:69-72.

18. Watson ST, Schaller TM, Tanner SL, Adams JD, Jeray KJ. Outcomes of low-energy basicervical proximal femoral fractures treated with cephalomedullary fixation. J Bone Joint Surg Am. 2016;98:1097-102.

19. Kweon SH, Lee SH, Kook SH, Choi YC. Outcomes of cephalomedullary nailing in basicervical fracture. Hip Pelvis. 2017;29:270-6.

20. Tasylkan L, Ugutmen E, Sanel S, Soylemez MS, Ozkan K, Solakoglu C. Short-term results of surgical treatment with cephalomedullary nails for basicervical proximal femoral fractures. Acta Orthop Belg. 2015;81:427-34.

21. Sawaguchi T, Sakagoshi D, Shima Y, Ito T, Goldhahn S. Do design adaptations of a trochanteric nail make sense for Asian patients? Results of a multicenter study of the PFNAII in Japan. Injury. 2014;45:1624-31.

22. Li J, Cheng L, Jing J. The Asia proximal femoral nail antirotation versus the standard proximal femoral antirotation nail for unstable intertrochanteric fractures in elderly Chinese patients. Orthop Traumatol Surg Res. 2015;101:143-6.

23. Broderick JM, Bruce-Brand R, Stanley E, Mulhall KJ. Osteoporotic hip fractures: the burden of fixation failure. ScientificWorldJournal. 2013;2013:515197.

24. Tsukada S, Okumura G, Matsueda M. Postoperative stability on lateral radiographs in the surgical treatment of pertrochanteric hip fractures. Arch Orthop Trauma Surg. 2012;132:839-46. 